\title{
Perfusion CT to Evaluate the Effect of Transluminal Angioplasty on Cerebral Perfusion in the Treatment of Vasospasm After Subarachnoid Hemorrhage
}

Charles B. L. M. Majoie,' Leonard J. van Boven,' Diederik van de Beek, 2,* Henk W. Venema,,3 and Willem J. van Rooij ${ }^{4}$

'Department of Radiology, ${ }^{2}$ Neurology, and ${ }^{3}$ Medical Physics, Academic Medical Center, University of Amsterdam, Amsterdam; the ${ }^{4}$ Department of Radiology, St. Elisabeth Hospital,Tilburg, the Netherlands

*Correspondence and Reprint Requests to:

Diederik van de Beek Department of Neurology Academic Medical Center, University of Amsterdam P.O. Box 22700, $1100 \mathrm{DE}$ Amsterdam, The Netherlands E-mail d.vandebeek@amc.uva.nl

\begin{abstract}
Introduction: Delayed ischemic neurologic deficits secondary to vasospasm are a major cause of morbidity and mortality after subarachnoid hemorrhage (SAH). Treatment of vasospasm after $\mathrm{SAH}$ is associated with complications, and reliable techniques for evaluating effects of treatment of vasospasm in such patients are warranted. We present the use of perfusion computed tomography (PTC) to evaluate the effect of transluminal percutaneous angioplasty in a with SAH and vasospasm-induced ischemia.
\end{abstract}

Methods: Dynamic PCT with deconvolution produced maps of time-to-peak, mean transit time, regional cerebral blood flow, and regional cerebral blood volume, with a computerized automated map of the infarct and penumbra. CT scanners with quadruple detector array were used before and after angioplasty.

Results: Before angioplasty and intraarterial papaverine, PCT showed normal to decreased cerebral blood flow and increased cerebral blood volume and mean transit time in the middle cerebral artery territory of the left hemisphere. After angioplasty and intraarterial papaverine, PCT showed normalization of perfusion parameters.

Conclusion: PCT can be a useful technique in monitoring angioplasty treatment effects in patients with vasospasm after SAH.

Key Words: Subarachnoid hemorrhage; perfusion CT; angioplasty; vasospasm.

(Neurocrit. Care 2007;06:40-44)

\section{Introduction}

Delayed ischemic neurologic deficits secondary to vasospasm are a major cause of morbidity and mortality after subarachnoid hemorrhage (SAH) (1). The development of vasospasm can be recognized by neurologic deterioration in combination with radiological techniques, such as conventional digital subtraction angiography (DSA), transcranial Doppler (TCD) ultrasound, positron emission tomography (PET), single-photon emission computer tomography (SPECT), and single-slice perfusion computed tomographic technique (Xe-CT) (1-6). Two recent studies showed that perfusion CT (PCT) also represents an accurate screening test in patients with suspected vasospasm, although further refinements are needed before routine recommendation $(6,7)$. Once symptomatic vasospasm is evident (with focal neurologic signs), hemodynamic augmentation is widely employed, although definitive evidence for this therapy is still lacking $(1,8,9)$. Patients whose condition does not improve 
with medical therapy undergo cerebral angiography and transluminal angioplasty (1,10-12). Aggressive treatment of vasospasm is not without risk (1,8). Hemodynamic augmentation has a complication rate of $10-20 \%$ and balloon angioplasty may be complicated by vessel rupture, branch occlusion, and hemorrhagic infarction (1,8-11). Therefore, accurate monitoring of treatment effects in patients with vasospasm after SAH is needed. We report the results of the use of PCT to evaluate the effect of transluminal percutaneous angioplasty and intraarterial papaverine in a patient with $\mathrm{SAH}$ and vasospasminduced ischemia.

\section{Case Report}

A 37-year-old woman was admitted with severe sudden headache, nausea, vomiting, and neck stiffness (Hunt \& Hess grade II). Unenhanced CT showed SAH predominantly in the left Sylvian fissure and over the left lateral convexity (Fisher grade 3). DSA showed a left internal carotid artery tip aneurysm, which was treated with endovascular coil placement (Guglielmi Detachable Coils, Boston Scientific, Cork, Ireland). After the procedure she had mild headache and no neurological deficits. On the fourth day after coil placement, however, she developed aphasia. TCD revealed severe vasospasm. Despite hemodynamic augmentation and nimodipine, she remained symptomatic and angioplasty was considered. PCT was performed 6 days after coil placement to determine if there was brain tissue at risk for cerebral infarction. Angioplasty was performed and PCT was repeated after angioplasty.

CT scanners with quadruple detector array were used before and after angioplasty. For PCT, we used a technique described previously $(3,4,7)$. In summary, after unenhanced CT of the brain, PCT was performed twice at two different levels, at the roof of the third ventricle and at the roof of the lateral ventricles. For each examination $40 \mathrm{~mL}$ of a nonionic contrast agent (iodixanol, Visipaque $320 \mathrm{mg}$ of iodine per $\mathrm{mL}$, GE Health, Cork, Ireland) were injected with a power injector into
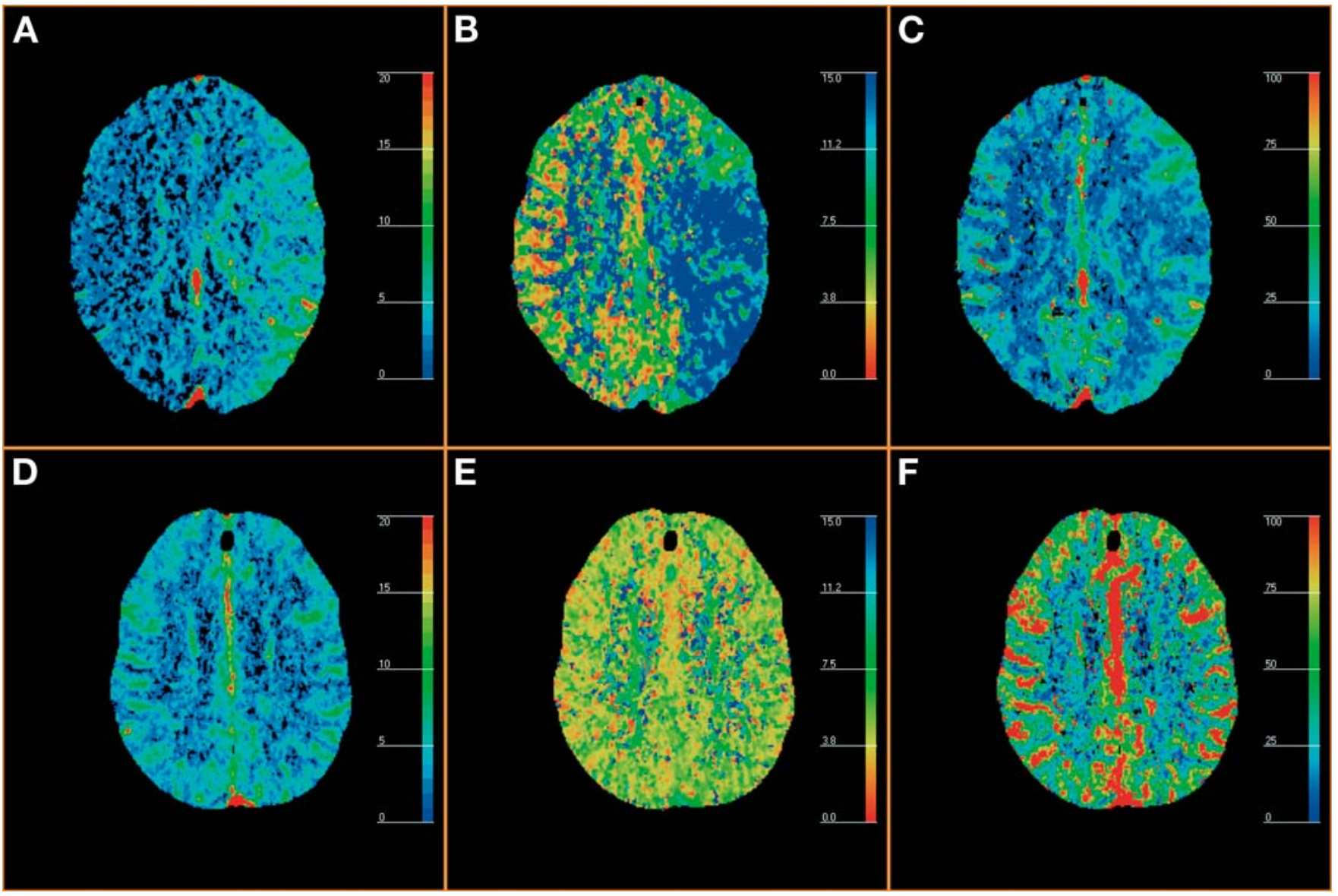

Fig. I. PCT was performed to determine if the patient was at risk for cerebral infarction and whether transluminal angioplasty should be performed or not (A-C) and was repeated after angioplasty to monitor treatment effects on cerebral perfusion (D-F). (A) Preangioplasty PCT CBV map demonstrates increased CBV values in the left hemisphere, consistent with ischemia-related activation of cerebral autoregulation (blue to red: $C B V=0$ to $20 \mathrm{~mL} / \mathrm{l00g}$ ). (B) Preangioplasty PCT MTT map demonstrates increased MTT values in the left hemisphere, consistent with ischemia-related activation of cerebral autoregulation (red to blue: MTT $=0$ to I5 seconds). (C) Preangioplasty PCT CBF map demonstrates normal to decreased CBF values in the left hemisphere (blue to red: $C B F=0$ to $100 \mathrm{~mL}$ per $100 \mathrm{~g}$ and per minute). (D) Postangioplasty PCT CBV map demonstrates normalized CBV values in the left hemisphere, symmetric to the normal right side. (E) Postangioplasty perfusion CT MTT map demonstrates normalized MTT values in the left hemisphere, symmetric to the normal right side. (F) Postangioplasty PCT CBF map demonstrates normalized CBF values in the left hemisphere, symmetric to the normal right side. 

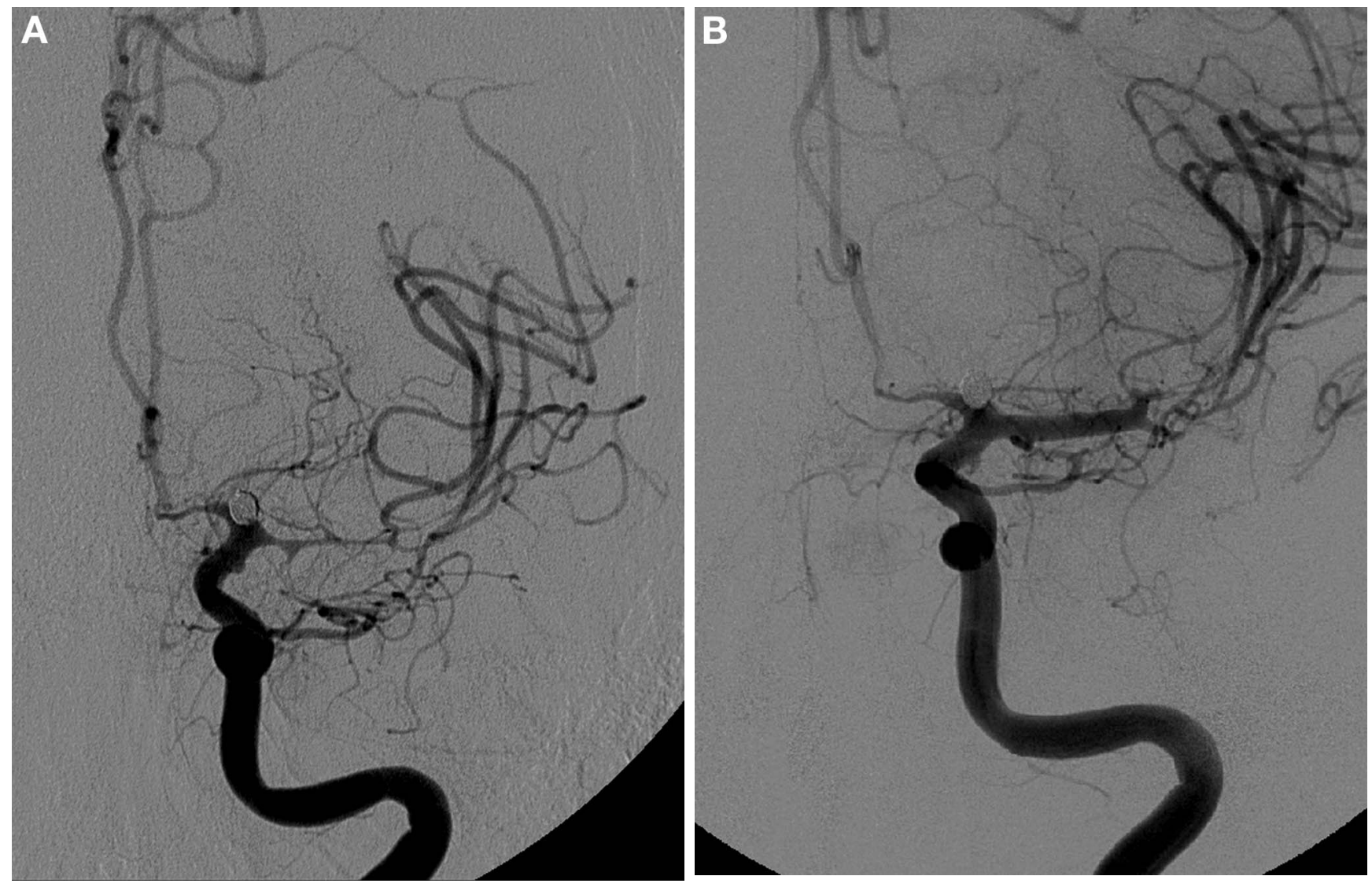

Fig. 2. DSA images in a 37-year-old woman who developed progressive aphasia I day after coiling of a ruptured left internal carotid arterytip aneurysm, despite optimal medical treatment. Transluminal balloon angioplasty was performed to treat the vasospasm. (A) Preangioplasty DSA demonstrates severe vasospasm in the MI and M2 segments of left MCA. (B) Postangioplasty DSA shows return to normal caliber of the MI-segment of the left MCA.

a cubital vein at a rate of $4 \mathrm{~mL} /$ second. At 7 seconds after initiation of the injection, a continuous scan was started with the following parameters: $80 \mathrm{kV}, 100 \mathrm{~mA}$ (Sensation 4) or $90 \mathrm{kV}$, $120 \mathrm{~mA}$ (Mx8000), $4 \times 5$-mm collimation, 1 second per rotation for 46 seconds. The four 5 -mm sections were reformatted into two 10-mm-thick sections. The overall dose required for PCT was $4.0-4.5 \mathrm{mSv}$. PCT data were analyzed with commercially available software developed by Wintermark et al. (14). Imagepostprocessing involved placement of the region of interest (ROI) in an input artery (anterior cerebral artery) and an input vein (torcular herophili or superior sagittal sinus) and subsequent generation of contrast-enhancement curves. The software generated color-coded cerebral blood flow $(\mathrm{CBF})$, cerebral blood volume (CBV) and mean transit time (MTT) maps based on the CT time attenuation curves for each pixel. After large vessel removal, four circular ROIs of $1.5 \mathrm{~cm}$ in diameter were placed along the cortical mantle of each hemisphere in the middle cerebral artery (MCA) territory on the perfusion maps to generate quantitative data. CBF, CBV, and MTT values in the normal right hemisphere were compared with those of the affected left hemisphere.

Unenhanced CT revealed a small left insular infarct. PCT showed areas of normal or decreased CBF and a large area of increased CBV and MTT in the MCA territory of the left hemi- sphere (Figure 1A-C), consistent with ischemia-related activation of autoregulation. The mean value of cortical CBF measured in the MCA territory of the right (normal) hemisphere was $77 \mathrm{~mL}$ per $100 \mathrm{~g}$ and per minute (range: 40-174). The mean value of cortical CBF in the left (affected) hemisphere was 31 $\mathrm{mL}$ per $100 \mathrm{~g}$ and per min (range: 13-38). The mean value of cortical CBV measured in the right (normal) hemisphere was $1.4 \mathrm{~mL} / 100 \mathrm{~g}$ (range: 1.1-1.8). The mean value of cortical CBV in the left (affected) hemisphere was $4.7 \mathrm{~mL} / 100 \mathrm{~g}$ (range 3.96.0). The mean value of cortical MTT measured in the right (normal) hemisphere was 1.2 seconds (range 0.6-1.9). The mean value of cortical MTT in the left (affected) hemisphere was 9.6 seconds (range 6.7-19.0).

On the same day as the PCT, angioplasty was performed under general anesthesia with full heparinization via a transfemoral approach using an over the wire (Transend 0.010 in, Target Therapeutics/Boston Scientific, Boston, MA) silicone balloon microcatheter (Sentry, Target Therapeutics/Boston Scientific, Fremont, CA), according to a technique described previously $(11,15)$. Before angioplasty, DSA showed severe vasospasm of the M1 segment of the left MCA and of the proximal left M2 segments (Figure 2A). After angioplasty of the M1 segment of the left MCA a good angiographic result was obtained (Figure 2B). In addition, $150 \mathrm{mg}$ of papaverine 
(papaverine hydrochloride, Eli Lilly and Co., Indianapolis, IN) was infused via a microcatheter in the left MCA to treat spasm of the proximal M2 segments. Within 2 days, her aphasia disappeared. PCT was repeated and showed symmetric, normalized CBV, CBF, and MTT values (Figure 1D-F).

\section{Case Discussion}

This case illustrates the value of PCT in demonstrating the effect of transluminal balloon angioplasty on cerebral perfusion in a patient with vasospasm after SAH. Before angioplasty, decreased to normal CBF and increased CBV and MTT were identified in the left MCA territory, indicating tissue at risk for cerebral infarction with intact autoregulation. After angioplasty, perfusion parameters returned to normal values and were symmetric.

Cerebral vasospasm is a frequent complication in patients with aneurysmal SAH (1). In $50 \%$ of cases it is manifested by delayed neurologic deficits related to ischemia, with progression to infarction in approximately one-half of the symptomatic cases $(1,8)$. Angioplasty for vasospasm may significantly reduce morbidity and mortality if performed early after medical therapy has failed $(1,10)$. It is, albeit rarely, also associated with severe complications, such as vessel rupture, and should therefore only be used in those patients in whom vasospasm causes cerebral ischemia $(1,8)$. Because delayed neurologic deficits following SAH may have different causes, including hydrocephalus, hemorrhage, edema, metabolic factors, and ischemia (12), it is important to identify those patients with vasospasm who most likely will benefit from angioplasty. Although TCD may aid in the determination of vasospasm, it does not necessarily assist in the identification of ischemia (12). SPECT (2), Xe-CT (3), and PET $(4,5)$ are reliable methods for the determination of cerebral perfusion, but these techniques are cumbersome and not widely available. PCT can be combined with unenhanced $\mathrm{CT}$, which is routinely performed for follow-up of patients with SAH (7). Nabavi et al. used this technique for monitoring cerebral perfusion after aneurysmal SAH (6). They found significant differences in CBF and CBV between patients with significant and absent or minor vasospasm and between patients with and without a presumed vasospasm-related infarct. They could not, however, define a PCT-threshold for cerebral infarct by using PCT, because of the use of relatively large ROIs, causing volume averaging with normal and ischemic tissue $(6,16)$.

The findings of the current report are consistent with results described by Grubb et al. (17). The system of autoregulation is reflected by PCT findings in patients with cerebral ischemia (18). A mild reduction in cerebral perfusion pressure is associated with a compensatory dilatation of cerebral blood vessels. Under these circumstances, measurements of $\mathrm{CBF}$ remain within normal range, while those of CBV and MTT increase. As perfusion pressure falls, normal CBF cannot be maintained, despite further vasodilatation (oligemia or misery perfusion). When perfusion pressure falls below a threshold of approximately $20 \mathrm{~mL}$ per $100 \mathrm{~g}$ and per minute, cerebral metabolism is severely impaired (ischemia). The development of subsequent irreversible damage (infarction) is not only dependent on the absolute perfusion value but also on the time the tissue has been ischemic. Irreversible damage is associated with loss of autoregulatory vasodilatation and therefore reduced CBV (18). PCT may also be valuable in detecting subclinical ischemia, i.e., areas of vasospasm which are still asymptomatic owing to intact autoregulation but may become symptomatic over time. Further research is warranted to determine if angioplasty of spastic vessels supplying these areas may improve outcome of patients with SAH.

PCT has several limitations, including restricted anatomic coverage and lack of full validation of its accuracy, reliability, and reproducibility of quantitative results $(7,16,19,20)$. With our multislice CT scanner, we were able to use a collimation of $4 \times 5 \mathrm{~mm}$ and to obtain $2 \times 10 \mathrm{~mm}$ reconstructed slices. By performing PCT at two different levels we could obtain $4 \mathrm{~cm}$ brain coverage. With 16-, 32- and 64-slice CT scanners, a larger brain coverage will be possible. Quantitation is strongly dependent on the choice of the venous ROI location $(19,20)$. Different ROI locations within the same vein may result in significantly different CBV and CBF values (19). The venous ROI with the greatest peak enhancement value should be chosen, as is done in the software used in this study to limit variability and increase quality of PCT images (19). With proper ROI selections a good correlation between quantitative PCT maps and xenon-CT and positron emission tomography (PET) studies was found, as described in previous reports $(3,5)$.

This case illustrates the value of PCT for monitoring treatment effects in patients with symptomatic vasospasm. This minimally invasive technique is widely available and requires only a modest radiation dose.

\section{Acknowledgments}

Diederik van de Beek received funding from the Meningitis Research Foundation UK, the Jan Meerwaldt Foundation, and the Netherlands Organization of Scientific Research (Rubicon); no conflicts of interest.

\section{References}

1 Suarez JI, Tarr RW, Selman WR. Aneurysmal subarachnoid hemorrhage. N Engl J Med 2006;354:387-396.

2 Lewis DH, Eskridge JM, Newell DW, et al. Brain SPECT and effect of cerebral angioplasty in delayed ischemia due to vasospasm. J Nucl Med 1992:33:1789-1796.

3 Wintermark M, Maeder P, Thiran J-Ph, et al. Simultaneous measurements of regional cerebral blood flows by perfusion-CT and stable xenon-CT: a validation study. AJNR Am J Neuroradiol 2001;22:905-914.

4 Powers WJ, Grubb RL, Baker RP, Mintun MA, Raichle ME. Regional cerebral blood flow and reversible ischemia due to vasospasm. Determination by positron emission tomography. J Neurosurg 1985;62:539-546.

5 Kudo K, Terae S, Katoh C, et al. Quantitative cerebral blood flow measurement with dynamic perfusion CT using the vascularpixel elimination method: comparison with $\mathrm{H} 2(15) \mathrm{O}$ positron emission tomography. AJNR Am J Neuroradiol 2003;24:419-426.

6 Nabavi DG, LeBlanc LM, Baxter B, et al. Monitoring cerebral perfusion after subarachnoid hemorrhage using CT. Neuroradiology 2001;43:7-16.

7 Wintermark M, Ko NU, Smith WS, Liu S, Higashida RT, Dillon WP. Vasospasm after subarachnoid hemorrhage: utility of perfusion CT and CT angiography on diagnosis and management. AJNR Am J Neuroradiol 2006;27:26-34.

8 Sen J, Belli A, Albon H, Morgan L, Petzold A, Kitchen N. Triple-H therapy in the management of aneurysmal subarchnoid haemorrhage. Lancet Neurology 2003;2:614-620.

9 Lee Kh, Lukovits T, Friedman JA. "Triple-H" therapy for cerebral vasospasm following subarachnoid hemorrhage. Neurocrit Care 2006;4:68-76. 
10 Higashida RT, Halbach VV, Dowd CF, Dormandy B, Bell J, Hieshima GB. Intravascular balloon dilatation therapy for intracranial arterial vasospasm: patient selection, technique and clinical results. Neurosurg Rev 1992;15:89-95.

11 Eskridge JM, McAuliffe W, Song JK, et al. Balloon angioplasty for the treatment of vasopasm: results of first 50 cases. Neurosurgery 1998;42:510-517.

12 Firlik AD, Kaufman AM, Jungreis CA, Yonas H. Effect of transluminal angioplasty on cerebral blood flow in the management of symptomatic vasospasm following aneurysmal subarachnoid hemorrhage. J Neurosurg 1997;86:830-839.

13 Wintermark M, Maeder P, Thiran JP, Schnyder P, Meuli R. Quantitative assessment of regional cerebral blood flows by perfusion $\mathrm{CT}$ studies at low injection rates: a critical review of the underlying theoretical models. Eur Radiol 2001;11: $1220-1230$.

14 Wintermark M, Fischbein NJ, Smith WS, Ko NU, Quist M, Dillon WP. Accuracy of dynamic perfusion CT with deconvolution in detecting acute hemispheric stroke. AJNR Am J Neuroradiol 2005;26:102-112.

15 Eskridge JM, Song JK. A practical approach to the treatment of vasospasm. AJNR Am J Neuroradiol 1997;18:1653-1660.

16 Hoeffner EG, Case I, Jain R, et al. Cerebral perfusion CT: technique and clinical applications. Radiology 2004;231:632-644.

17 Grubb RL, Raichle ME, Eichling JO, Gado MH. Effects of subarachnoid hemorrhage on cerebral blood volume, blood flow and oxygen utilization in humans. J Neurosurg 1977;46:446-453.

18 Miles KA, Griffiths MR. Perfusion CT: a worthwile enhancement? Brit J Radiol;76:220-231.

19 Kealey SM, Loving VA, Delong DM, Eastwood JD. User-defined vascular input function curves: influence on mean perfusion parameter values and signal-to-noise ratio. Radiology 2004;231: 587-593.

20 Sanelli PC, Lev MH, Eastwood JD, Gonzalez RG, Lee TY. The effect of varying user-selected input parameters on quantitative values in CT perfusion maps. Acad Radiol 2004;11:1085-1092. 